\title{
Collapse of solitary excitations in the nonlinear Schrödinger equation with nonlinear damping and white noise
}

Christiansen, Peter Leth; Gaididei, Yuri Borisovich; Rasmussen, Kim; Johansson, Magnus; Yakimenko, Irina $\mathbf{~}$.

Published in:

Physical Review E. Statistical, Nonlinear, and Soft Matter Physics

Link to article, DOI:

10.1103/PhysRevE.54.924

Publication date:

1996

Document Version

Publisher's PDF, also known as Version of record

Link back to DTU Orbit

Citation (APA):

Christiansen, P. L., Gaididei, Y. B., Rasmussen, K., Johansson, M., \& Yakimenko, I. I. (1996). Collapse of solitary excitations in the nonlinear Schrödinger equation with nonlinear damping and white noise. Physical Review E. Statistical, Nonlinear, and Soft Matter Physics, 54(1), 924-930.

https://doi.org/10.1103/PhysRevE.54.924

\section{General rights}

Copyright and moral rights for the publications made accessible in the public portal are retained by the authors and/or other copyright owners and it is a condition of accessing publications that users recognise and abide by the legal requirements associated with these rights.

- Users may download and print one copy of any publication from the public portal for the purpose of private study or research.

- You may not further distribute the material or use it for any profit-making activity or commercial gain

- You may freely distribute the URL identifying the publication in the public portal 


\title{
Collapse of solitary excitations in the nonlinear Schrödinger equation with nonlinear damping and white noise
}

\author{
Peter L. Christiansen, ${ }^{1}$ Yuri B. Gaididei, ${ }^{2}$ Magnus Johansson, ${ }^{1}$ Kim $\emptyset$. Rasmussen, ${ }^{1}$ and Irina I. Yakimenko ${ }^{2}$ \\ ${ }^{1}$ Institute of Mathematical Modelling, The Technical University of Denmark, DK-2800 Lyngby, Denmark \\ ${ }^{2}$ Institute forTheoretical Physics, Metrologicheskaya Street 14 B, 252143 Kiev 143, Ukraine
}

(Received 14 November 1995)

\begin{abstract}
We study the effect of adding noise and nonlinear damping in the two-dimensional nonlinear Schrödinger equation (NLS). Using a collective coordinate approach, we find that for initial conditions where total collapse occurs in the unperturbed NLS, the presence of the damping term will instead result in an exponentially decreasing width of the solution in the long-time limit. We also find that a sufficiently large noise variance may cause an initially localized distribution to spread instead of contracting, and that the critical variance necessary to cause dispersion will for small damping be the same as for the undamped system. [S1063-651X(96)11207-1]
\end{abstract}

PACS number(s): 03.40.kf, 05.40.+j

\section{INTRODUCTION}

The two-dimensional (2D) nonlinear Schrödinger equation (NLS) can be obtained, e.g., in a continuum approximation of a model for 2D exciton-phonon coupled systems, which has been used to model, for example, the Scheibe molecular aggregates [1]. It is well known [2] that for some initial conditions, the 2D NLS exhibits blowup; i.e., the maximum of the solution will tend to infinity in a finite time. In the case where the total "mass" of the wave collapses into one single point, total collapse is said to occur, and the time when this happens is termed the collapse time. If thermal fluctuations of the phonon system are taken into account, the resulting continuum equation for the excitons was shown [3] to include a term containing colored multiplicative noise. Approximating the complicated noise spectrum with Gaussian white noise, it was shown [4] that the presence of noise would delay the collapse process, and that the collapse could be destroyed if the variance of the noise was sufficiently large.

However, to allow the exciton system to reach thermal equilibrium, the noise term providing energy input to the exciton system should be balanced by another term providing energy dissipation. In this paper we suggest that this balance can be obtained by adding a nonlinear damping term to the equation. Nonlinear damping in NLS systems has previously also been considered in the context of nonlinear optics $[5,6]$. We use a collective coordinate approach to derive an ordinary differential equation for the width of the trial exciton wave function. Solving this equation numerically we find that blowup will not occur when the damping term is included; the solution will be well defined for all times but its width will approach zero exponentially in the absence of noise. This behavior, which seems physically more reason- able than a true collapse, will be termed "pseudocollapse." As long as the damping is not too large, the critical variance of the noise required to cause dispersion instead of contraction is found to be approximately the same as for the case without damping.

\section{MODEL}

Following the derivation given in Ref. [3], we start by assuming that the coupled exciton-phonon system can be described by the following pair of equations:

$$
\begin{aligned}
i \hbar \dot{\psi}_{n}+\sum_{n^{\prime}} J_{n n^{\prime}} \psi_{n^{\prime}}+\chi u_{n} \psi_{n} & =0, \\
M \ddot{u}_{n}+M \lambda \dot{u}_{n}+M \omega_{0}^{2} u_{n}-\chi\left|\psi_{n}\right|^{2} & =\eta_{n}(t) .
\end{aligned}
$$

Here $\psi_{n}$ is the amplitude of the exciton wave function corresponding to site $n$ and $u_{n}$ represents the elastic degree of freedom at site $n$. Furthermore, $-J_{n n^{\prime}}$ is the dipole-dipole interaction energy, $\chi$ is the exciton-phonon coupling constant, $M$ is the molecular mass, $\lambda$ is the damping coefficient, $\omega_{0}$ is the Einstein frequency of each oscillator, and $\eta_{n}(t)$ is an external force acting on the phonon system. To describe the interaction of the phonon system with a thermal reservoir at temperature $T, \eta_{n}(t)$ is assumed to be Gaussian white noise with zero mean and the autocorrelation function

$$
\left\langle\eta_{n}(t) \eta_{n^{\prime}}\left(t^{\prime}\right)\right\rangle=2 M \lambda k_{B} T \delta\left(t-t^{\prime}\right) \delta_{n n^{\prime}},
$$

in accordance with the fluctuation-dissipation theorem ensuring thermal equilibrium.

In order to derive a single equation for the dynamics of the exciton system, we start by writing the solution to Eq. (2) in the integral form

$$
u_{n}(t)=u_{n}^{(0)}(t)+\frac{1}{M\left(S_{+}-S_{-}\right)} \int_{0}^{t} d t^{\prime}\left(e^{S_{+}\left(t-t^{\prime}\right)}-e^{S_{-}\left(t-t^{\prime}\right)}\right)\left[\chi\left|\psi_{n}\left(t^{\prime}\right)\right|^{2}+\eta_{n}\left(t^{\prime}\right)\right],
$$

where 


$$
S_{ \pm}=-\lambda / 2 \pm \sqrt{(\lambda / 2)^{2}-\omega_{0}^{2}}
$$

and

$$
u_{n}^{(0)}(t)=\frac{1}{S_{+}-S_{-}}\left\{\left(\lambda u_{n}(0)+\dot{u}_{n}(0)\right)\left(e^{S_{+} t}-e^{S_{-} t}\right)+u_{n}(0)\left(S_{+} e^{S_{+} t}-S_{-} e^{S_{-} t}\right)\right\}
$$

Here $u_{n}(0)$ and $\dot{u}_{n}(0)$ are the molecular displacements and velocities at the initial moment of time. From Eq. (6) we observe that $u_{n}^{(0)}(t)$ will decay as $e^{-(\lambda / 2) t}$, and since we will be interested in times such that $t \gg 2 / \lambda$ we can neglect this transient term in Eq. (4). Furthermore, using repeated partial integration we can write

$$
\int_{0}^{t} d t^{\prime} e^{S_{ \pm}\left(t-t^{\prime}\right)}\left|\psi_{n}\left(t^{\prime}\right)\right|^{2}=\left[-e^{S_{ \pm}\left(t-t^{\prime}\right)}\left(\frac{1}{S_{ \pm}}\left|\psi_{n}\left(t^{\prime}\right)\right|^{2}+\frac{1}{S_{ \pm}^{2}} \frac{d}{d t^{\prime}}\left[\left|\psi_{n}\left(t^{\prime}\right)\right|^{2}\right]\right)\right]_{t^{\prime}=0}^{t^{\prime}=t}+\frac{1}{S_{ \pm}^{2}} \int_{0}^{t} d t^{\prime} e^{S_{ \pm}\left(t-t^{\prime}\right)} \frac{d^{2}}{d t^{\prime 2}}\left[\left|\psi_{n}\left(t^{\prime}\right)\right|^{2}\right] .
$$

The last term in Eq. (7) can be neglected if $\left|\psi_{n}(t)\right|^{2}$ is assumed to vary sufficiently slowly in time compared with the lattice vibrations, i.e., if

$$
\frac{\omega_{0}^{2}-(\lambda / 2)^{2}}{\omega_{0}^{4}} \frac{1}{\left|\psi_{n}(t)\right|^{2}} \frac{d^{2}}{d t^{2}}\left[\left|\psi_{n}(t)\right|^{2}\right] \ll 1
$$

Neglecting all exponentially decaying transient terms, we thus obtain an approximate expression for the molecular displacements from Eqs. (4), (5), and (7):

$$
u_{n}(t) \approx \frac{\chi}{M \omega_{0}^{2}}\left(\left|\psi_{n}(t)\right|^{2}-\frac{\lambda}{\omega_{0}^{2}} \frac{d}{d t}\left[\left|\psi_{n}(t)\right|^{2}\right]\right)+\sigma_{n}(t) .
$$

Here $\sigma_{n}(t)$, defined as

$$
\sigma_{n}(t)=\frac{1}{M\left(S_{+}-S_{-}\right)} \int_{0}^{t} d t^{\prime}\left(e^{S_{+}\left(t-t^{\prime}\right)}-e^{S_{-}\left(t-t^{\prime}\right)}\right) \eta_{n}\left(t^{\prime}\right),
$$

is a new stochastic force described by noise that is not white, but strongly colored [3].

Introducing the expression (9) for the molecular displacements into Eq. (1), we immediately get the following equation involving only exciton variables:

$$
\begin{aligned}
i \hbar \dot{\psi}_{n} & +\sum_{n^{\prime}} J_{n n^{\prime}} \psi_{n^{\prime}}+V\left|\psi_{n}\right|^{2} \psi_{n}-V \frac{\lambda}{\omega_{0}^{2}} \psi_{n} \frac{d}{d t}\left[\left|\psi_{n}(t)\right|^{2}\right] \\
& +\chi \sigma_{n}(t) \psi_{n}=0 .
\end{aligned}
$$

Here we have introduced the nonlinearity parameter $V$ defined as

$$
V=\chi^{2} / M \omega_{0}^{2}
$$

Note that the main difference between the derivation above and the one used in Ref. [3] is that we retain one more term in the expansion (7), the result of which is the presence of the nonlinear damping term $-V\left(\lambda / \omega_{0}^{2}\right) \psi_{n}(d / d t)\left[\left|\psi_{n}(t)\right|^{2}\right]$ in the exciton equation (11).

Making the additional assumptions that $\psi_{n}$ varies slowly in space and that only nearest-neighbour coupling $J$ is of importance, we obtain in the continuum approximation for the continuous exciton field $\psi(x, y, t)=e^{-i 4 J t / \hbar} \psi_{n}(t) / l$ :

$$
i \hbar \psi_{t}+J l^{2} \nabla^{2} \psi+V l^{2}|\psi|^{2} \psi-V \frac{\lambda}{\omega_{0}^{2}} l^{2} \psi\left(|\psi|^{2}\right)_{t}+\chi l^{2} \sigma \psi=0,
$$

where $l$ is the distance between nearest neighbors and $\sigma(x, y, t)=\sigma_{n}(t) / l^{2}$ is the noise density. Equation (13) can be cast into a more convenient form by transforming into dimensionless variables,

$$
\frac{x}{l} \rightarrow x, \quad \frac{y}{l} \rightarrow y, \quad J t / \hbar \rightarrow t, \quad \sqrt{\frac{V l^{2}}{J}} \psi \rightarrow \psi, \quad \frac{\chi l^{2}}{J} \sigma \rightarrow \sigma,
$$

which leads to

$$
i \psi_{t}+\nabla^{2} \psi+|\psi|^{2} \psi-\Lambda \psi\left(|\psi|^{2}\right)_{t}+\sigma \psi=0,
$$

where the nonlinear damping parameter $\Lambda$ is given by

$$
\Lambda=\lambda J / \hbar \omega_{0}^{2} .
$$

It may be interesting to note that an equation similar to the one-dimensional version of Eq. (15), but with $\left(|\psi|^{2}\right)_{t}$ replaced by $\left(|\psi|^{2}\right)_{x}$, is often encountered in the context of nonlinear optics, where it governs the effect of Raman scattering [5]. The presence of the term $\left(|\psi|^{2}\right)_{x}$ was recently shown to arrest the collapse of one-dimensional pulses in a NLS with critical nonlinearity [6].

It can be shown easily that in spite of the presence of the nonlinear damping and multiplicative noise terms in Eq. (15), the norm, defined as

$$
N=\iint|\psi(x, y, t)|^{2} d x d y,
$$

will still be a conserved quantity, having the value $N=V / J$ if the exciton wave function is assumed to be normalized in the physical coordinates. By writing $\psi=\sqrt{n} e^{i \theta}$, the following equations for the amplitude and phase of the solution can be obtained from (15): 


$$
\begin{gathered}
\frac{1}{2} n_{t}+\nabla(n \nabla \theta)=0, \\
-\theta_{t}-\Lambda n_{t}-(\nabla \theta)^{2}+n+\frac{1}{\sqrt{n}} \nabla^{2}(\sqrt{n})+\sigma(x, y, t)=0 .
\end{gathered}
$$

The norm conservation is immediately seen from the first of these equations, while the second equation shows that the role of the damping term is to destroy the phase coherence of the solution and cause a diffusionlike behavior for the phase. The ordinary NLS Hamiltonian, defined as

$$
H=\iint\left[|\nabla \psi(x, y, t)|^{2}-\frac{1}{2}|\psi(x, y, t)|^{4}\right] d x d y,
$$

will then in general no longer be conserved. Instead we find that

$$
\frac{d H}{d t}=\iint \sigma(x, y, t)\left(|\psi|^{2}\right)_{t} d x d y-\Lambda \iint\left[\left(|\psi|^{2}\right)_{t}\right]^{2} d x d y .
$$

Thus, the two terms provide energy input and energy dissipation to the exciton system, making an energy balance possible. Consequently, there is a possibility for the system to reach thermal equilibrium.

\section{COLLECTIVE COORDINATE APPROACH}

To investigate the influence on the collapse process of the damping and noise terms in Eq. (15), we will use the method of collective coordinates. To this end, we will make some simplifying assumptions. We will assume isotropy, which effectively reduces the problem to one space dimension with the radial coordinate $r=\sqrt{x^{2}+y^{2}}$. We also assume that the noise $\sigma$ can be approximated by radially isotropic Gaussian white noise with autocorrelation function

$$
\left\langle\sigma(r, t) \sigma\left(r^{\prime}, t^{\prime}\right)\right\rangle=\frac{D_{r}}{r} \delta\left(r-r^{\prime}\right) \delta\left(t-t^{\prime}\right),
$$

where $D_{r}$ is the dimensionless noise variance. The validity of this approximation was discussed in Ref. [3]. Finally, we assume that the collapse process can be described in terms of collective coordinates by using a localized self-similar trial function for the exciton wave function $\psi(r, t)$ of the form

$$
\psi(r, t)=A(t) f(r / B(t)) e^{i \alpha(t) r^{2}},
$$

where $f(x)$ is an arbitrary well-behaved, real function, which decreases sufficiently fast (e.g., exponentially) as $x \rightarrow \infty$. The three real time-dependent parameters $A, B$, and $\alpha$ thus determine the amplitude, width, and phase of the wave function, respectively. In Refs. [4] and [7], where the case $\Lambda=0$ in Eq. (15) was investigated, the particular choice $f(x)$ $=\operatorname{sech}(x)$ was considered. This choice was motivated by regarding it as a generalization of the approximate groundstate solution to the ordinary 2D NLS found in Ref. [8]. As long as $\Lambda$ is not too large, one might expect this trial function to give a good description of the collapse process also in the presence of damping, but since the qualitative features of the results obtained below do not depend on the explicit choice of trial function, the function $f$ will here be left unspecified [9].

From the definition (17) of the norm, with $\psi$ given by (22), we immediately obtain the relation between amplitude and width,

$$
A(t)=\frac{\sqrt{N / s_{1,2,0}},}{B(t)}
$$

where the coefficient $s_{1,2,0}$ is obtained from the general definition of the integrals $s_{m, n, p}$ :

$$
s_{m, n, p}=2 \pi \int_{0}^{\infty} r^{m}[f(r)]^{n}\left[f^{\prime}(r)\right]^{p} d r .
$$

In [7] a variational approach was used, and from the Euler-Lagrange equations the relation

$$
\alpha(t)=\frac{\dot{B}(t)}{4 B(t)}
$$

was obtained together with an ordinary differential equation for $B(t)$. However, in the presence of damping $(\Lambda \neq 0)$ this technique is not applicable. Instead, we will use the trial function (22) with $\alpha(t)$ given by (25) and derive an ordinary differential equation for $B(t)$ using the virial theorem. Defining the virial coefficient $W$ as

$$
W(t) \equiv \int \vec{r}^{2}|\psi(\vec{r}, t)|^{2} d \vec{r}=2 \pi \int_{0}^{\infty} r^{3}|\psi(r, t)|^{2} d r,
$$

we obtain from (15) that it satisfies the equation

$$
\begin{aligned}
\frac{1}{4} \frac{d^{2} W}{d t^{2}}= & 2 H-2 \pi \Lambda \int_{0}^{\infty} r^{2}|\psi|^{2} \partial_{r}\left[\partial_{t}\left(|\psi|^{2}\right)\right] d r \\
& +2 \pi \int_{0}^{\infty} r^{2}|\psi|^{2} \partial_{r}(\sigma) d r
\end{aligned}
$$

where $H$ is the Hamiltonian (19). Using (22)-(25), we arrive at the following differential equation for the width $B$ of the exciton wave function:

$$
\begin{aligned}
B^{3} \ddot{B}= & \Delta-\Gamma \frac{\dot{B}}{B}-\frac{8 \pi}{s_{3,2,0}} \int_{0}^{\infty}\left(1+\frac{r}{B} \frac{f^{\prime}(r / B)}{f(r / B)}\right) \\
& \times\left[f\left(\frac{r}{B}\right)\right]^{2} \sigma(r, t) r d r,
\end{aligned}
$$

where the constants $\Delta$ and $\Gamma$ are defined as

$$
\Delta=\frac{4}{s_{3,2,0}}\left(s_{1,0,2}-\frac{N s_{1,4,0}}{2 s_{1,2,0}}\right),
$$

and

$$
\Gamma=\frac{8 N \Lambda s_{3,2,2}}{s_{1,2,0} s_{3,2,0}},
$$

respectively. Note that $\Delta$ and $\Gamma$ depend on the initial conditions via $N$, and that while $\Delta$ can be either positive or nega- 
tive, $\Gamma$ is always positive. In the absence of noise and damping, it is evident from (28) that collapse will occur if and only if $\Delta<0$.

In analogy with the treatment for the undamped case in Ref. [7], we find that it is possible to transform Eq. (28) into a simpler stochastic differential equation without changing the Fokker-Planck equation for the system. This equation, which therefore gives an equivalent description of the process, gets the form

$$
\ddot{B}=\frac{\Delta}{B^{3}}-\frac{\Gamma \dot{B}}{B^{4}}+\frac{h(t)}{B^{2}},
$$

where $h(t)$ is white noise with the autocorrelation

$$
\left\langle h(t) h\left(t^{\prime}\right)\right\rangle=2 D \delta\left(t-t^{\prime}\right)
$$

Here the parameter $D$ giving the variance of $h(t)$ is related to the variance $D_{r}$ of $\sigma(r, t)$ defined in (21) through

$$
D=\frac{32 \pi^{2} D_{r} s_{3,2,2}}{s_{3,2,0}^{2}} .
$$

\section{NUMERICAL SOLUTION OF THE COLLECTIVE COORDINATE EQUATION}

In this section we analyze the influence of damping and noise on the collapse process by solving the ordinary stochastic differential equation (31) for the width $B$ of the trial function (22) numerically. The numerical solutions have been obtained using a stochastic version of the fourth-order Runge-Kutta-Merson algorithm [10] using its standard error estimate to adapt the size of the time steps so that the error of the deterministic part of the solution is controlled. To control the error of the stochastic part of the solution, we have checked that its statistical properties remain invariant when changing the maximum allowed time step and varying the sample size. We note that the algorithm used in general will give the Stratonovich solution to a stochastic differential equation [11], but that for our equation (31) this will be identical to the Ito solution. Thus, there is no ambiguity concerning the interpretation of the solution in this case.

With neither damping nor noise in the system $(\Gamma=D=0)$ and $\Delta<0$, the well-known exact solution

$$
B(t)=B_{0} \sqrt{1-t^{2} / t_{c}^{2}}, \quad t_{c} \equiv \frac{B_{0}^{2}}{\sqrt{|\Delta|}},
$$

fulfilling the initial conditions

$$
B(0)=B_{0}, \quad \dot{B}(0)=0
$$

is easily obtained. Thus, the solution collapses and ceases to exist at the collapse time $t=t_{c}$. When the damping term is present in Eq. (31), we find that strictly speaking no collapse will occur, since the solution will be well defined for all $t$. Instead we find that $B(t)$ will go exponentially to zero for large $t$,

$$
B(t) \sim e^{-(|\Delta| / \Gamma) t}, \quad t \rightarrow \infty,
$$

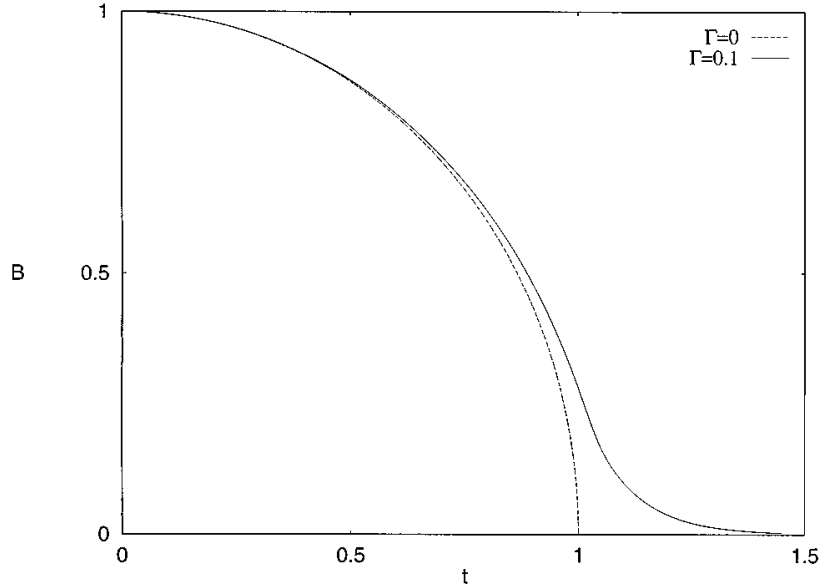

FIG. 1. Width $B$ as a function of time $t$ in the absence of noise. Dashed line shows the analytical solution (34) for $\Gamma=0$ with $B_{0}=|\Delta|=1$; solid line the numerical solution of Eq. (31) for $\Gamma=0.1$.

so that the process can be considered as a collapse process with an infinite collapse time. This type of behavior will be called "pseudocollapse.' The difference between the damped and the undamped cases is illustrated in Fig. 1. As can be seen, the initial stages of the pseudocollapse process will resemble a pure collapse as long as the damping is small. One can then roughly define a "pseudocollapse time", as the time where the asymptotic behavior (36) sets in.

In the presence of noise, but no damping, it was found in Ref. [4] that the collapse time would increase with increasing noise variance, and that the collapse process could be stopped if the variance was large enough. In this case, by rescaling the time variable

$$
\widetilde{t}=\sqrt{|\Delta|} t
$$

Eq. (31) with $\Gamma=0$ can be rewritten as

$$
\frac{d^{2} B}{d \widetilde{t}^{2}}=-\frac{1}{B^{3}}+\frac{\widetilde{h}(\widetilde{t})}{B^{2}},
$$

where the rescaled noise $\widetilde{h}(\widetilde{t})$ is still white but with the variance parameter $\widetilde{D}$ rescaled as

$$
\widetilde{D}=\frac{D}{|\Delta|^{3 / 2}}
$$

Thus, for given initial conditions $B(0)$ and $\dot{B}(0)$ and arbitrary $\Delta<0$, the critical variance $D$ required to stop the collapse will depend only on the parameter $\widetilde{D}$ given by Eq. (39).

When damping is also present, it is necessary to rescale not only $t$, but also $B$, in order to eliminate both the $\Delta$ and $\Gamma$ dependence from Eq. (31). The transformation will in this case be given by

$$
\tilde{t}=\frac{|\Delta|}{\Gamma} t, \quad \widetilde{B}=\frac{|\Delta|^{1 / 4}}{\sqrt{\Gamma}} B,
$$

while the variance of the rescaled noise still will be given by Eq. (39). Thus, disregarding the changes in time scale, the process is determined by two quantities, $\widetilde{D}$ from (39) and the 

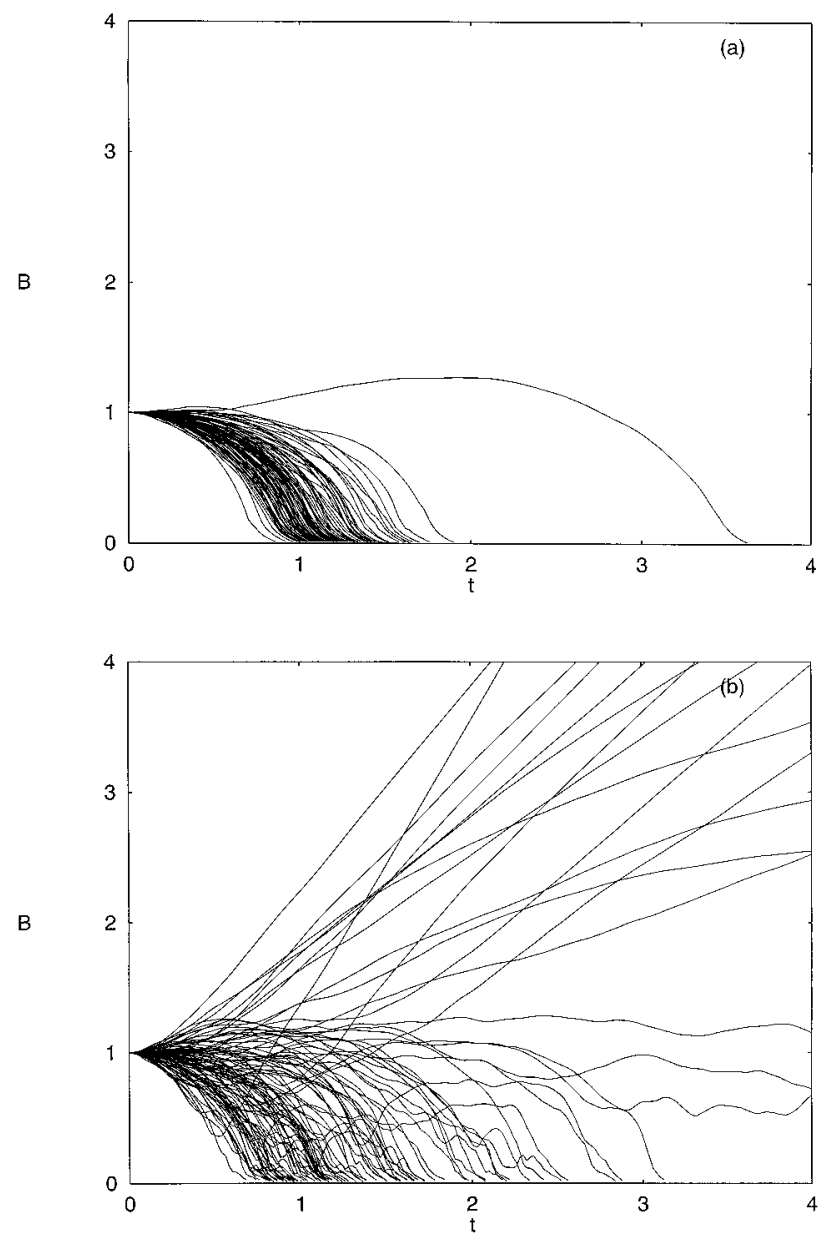

FIG. 2. Width $B$ as a function of time $t$ for 100 different configurations of the noise for $\Gamma=0.1$ and $D=$ (a) 0.05 and (b) 0.5. In both cases, $B_{0}=|\Delta|=1$. For computational reasons the numerical integration for each trajectory has been aborted when $B<0.01$, so that the exponential decrease towards zero as $t \rightarrow \infty$ is not shown.

initial value at $t=0$ of the quantity $\widetilde{B}$ as defined in (40). Consequently, an increase of the damping parameter $\Gamma$ will be equivalent to a decrease of the initial width $B_{0}$, and one would therefore expect that as long as $\Gamma$ is small compared to $|\Delta|^{1 / 2}$ the process will be practically indistinguishable from the undamped process except when $B \approx 0$, i.e., close to collapse.

To illustrate how the noise affects the (pseudo)collapse process, we show in Figs. 2(a) and 2(b) how the width $B$ varies with time $t$ for a sample of 100 different noise configurations and small damping $[\widetilde{B}(0) \approx 3.16]$. In (a), where $\widetilde{D}=0.05$, we see that all trajectories will finally enter the region of exponential decrease towards zero, but that the noise will cause a spread in the pseudocollapse time, defined as above as the approximate time where the asymptotic behaviour sets in. In (b), however, where $\widetilde{D}=0.5$, the noise is sufficiently strong to cause divergence of some trajectories. A more careful study shows that for this value of $\widetilde{B}(0)$, a value of $\widetilde{D} \approx 0.15$ is sufficient to cause divergence for more than $1 / 1000$ of the trajectories. To the accuracy we can obtain, this critical value of $\widetilde{D}$ is the same as for the undamped case $(\Gamma=0)$. However, for stronger damping [or, equivalently, smaller values of $\widetilde{B}(0)]$, even the initial stages of the pseudocollapse process will be affected by the damping, and
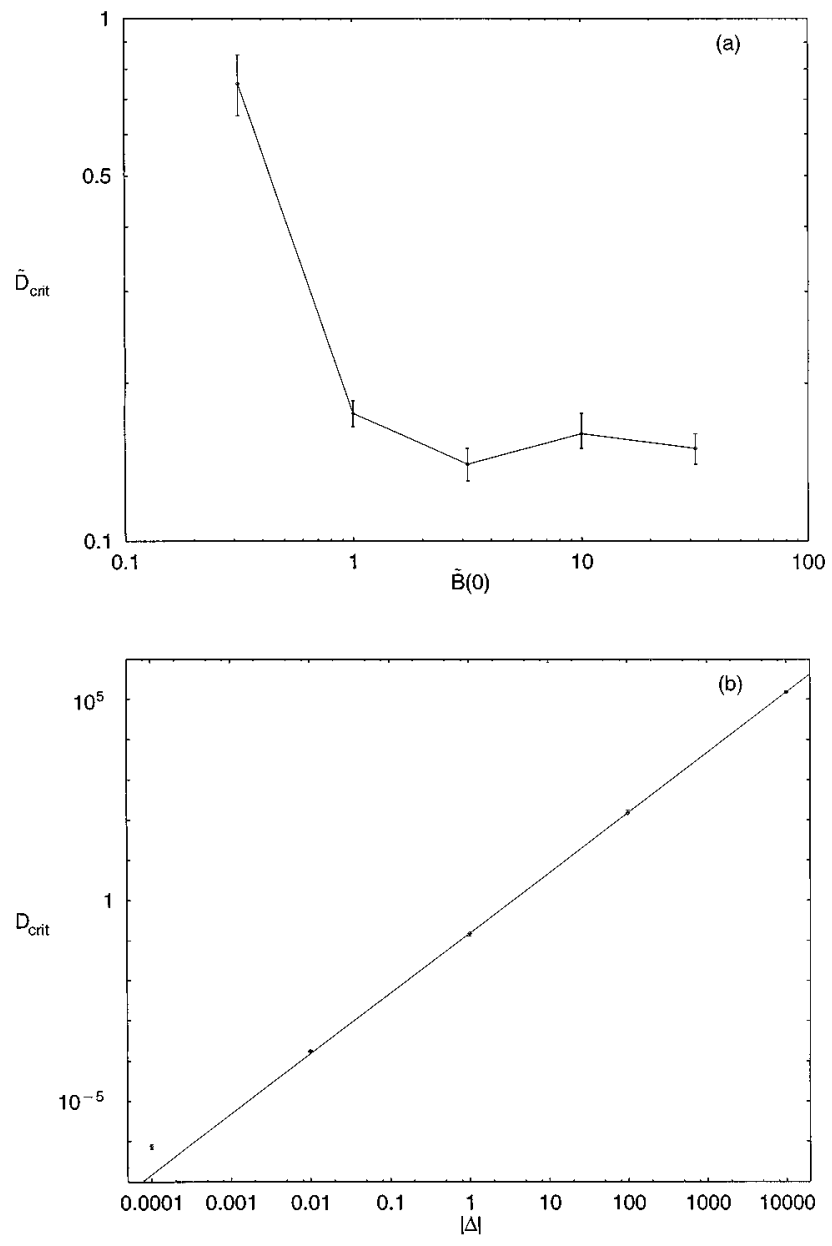

FIG. 3. (a) Critical value of $\widetilde{D}, \widetilde{D_{\text {crit }}}$, from Eq. (39) vs $\widetilde{B}(0)$ from Eq. (40). $\widetilde{D}_{\text {crit }}$, is here defined as the smallest value of $\widetilde{D}$ for which more than $1 / 1000$ of the trajectories obtained for different noise configurations diverge. The error bars correspond to results obtained for different samples. The line is a guide to the eye. (b) Critical value of $D, D_{\text {crit }}$, as a function of $|\Delta|$ when $\Gamma=0.1$ and $B_{0}=1$. The line is a plot of the function $D_{\text {crit }}=0.15|\Delta|^{3 / 2}$, which describes the scaling behavior in the absence of damping.

we find that the critical value of $\widetilde{D}$ in these cases will be increased. This is illustrated in Fig. 3(a), where the critical value of $\widetilde{D}, \widetilde{D}_{\text {crit }}$, is plotted as a function of $\widetilde{B}(0)$. In Fig. 3 (b) we plot the critical value of $D, D_{\text {crit }}$, as a function of $|\Delta|$ for the particular values $B_{0}=1$ and $\Gamma=0.1$ to illustrate that as long as $|\Delta|$ is large compared to $\Gamma^{2}$, the scaling $D_{\text {crit }}$ $\sim|\Delta|^{3 / 2}$ found in Ref. [4] for the undamped case will be valid also in the presence of damping.

A quantity of physical interest is the ensemble average of the width, $\langle B(t)\rangle$, since it is related to the mean value of the radial probability current $j_{r}(t)$ for the exciton system defined as

$$
\left\langle j_{r}(t)\right\rangle=\left\langle\frac{i}{2} \iint r d r d \theta\left(\psi \partial_{r} \psi^{*}-\psi^{*} \partial_{r} \psi\right)\right\rangle
$$

Using (22)-(24), the relation

$$
\left\langle j_{r}(t)\right\rangle=\frac{N s_{2,2,0}}{2 s_{1,2,0}}\langle\dot{B}(t)\rangle
$$




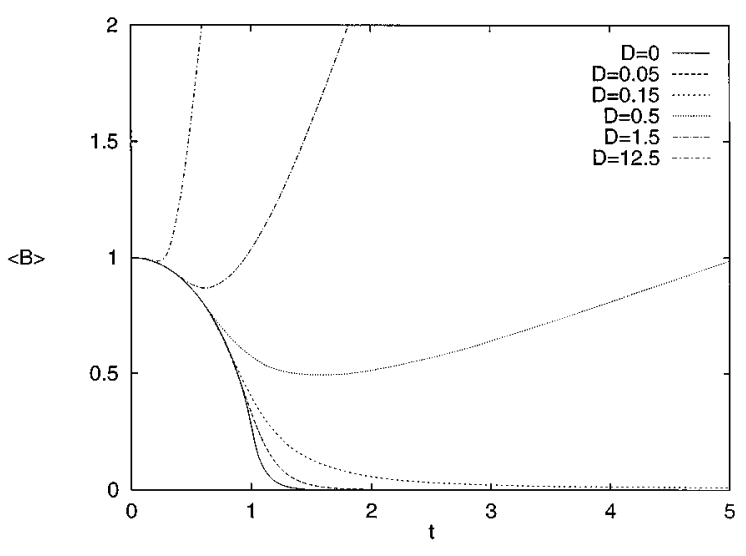

FIG. 4. Ensemble average of the width $\langle B\rangle$ as a function of time $t$ for different noise variances. From bottom to top we have $D=0$, $0.05,0.15,0.5,1.5$, and 12.5 , respectively. In all cases $B_{0}=|\Delta|=1$ and $\Gamma=0.1$.

is readily obtained. The behavior of $\langle B(t)\rangle$ is shown in Fig. 4 for the parameter values $B_{0}=|\Delta|=1, \Gamma=0.1$, and different values of the noise variance. It can be seen that for $D<D_{\text {crit }} \approx 0.15$, the effect of the noise is to delay the pseudocollapse in terms of the ensemble average of the width, in analogy with the similar result obtained in Ref. [4] for the undamped case. For $D>D_{\text {crit }}$, we observe a nonmonotonic behavior of $\langle B(t)\rangle$. Initially, the average width will decrease in a similar way as when $D<D_{\text {crit }}$, but after some time the dominating contribution to the mean value will come from the diverging trajectories, and $\langle B(t)\rangle$ will consequently also diverge as $t \rightarrow \infty$. There will thus be a minimum average width, which will increase towards $B_{0}$ as $D$ increases, as can be seen in Fig. 4.

An interesting question regarding the system described by the collective variable equation (31) is whether it is possible to obtain a balance between energy input and energy dissipation also in this simple approximation. Defining the energy as

$$
E=\frac{1}{2}\left(\dot{B}^{2}-\frac{|\Delta|}{B^{2}}\right),
$$

the following relation can be derived:

$$
\frac{d}{d t}\langle E\rangle=-\Gamma\left\langle\frac{\dot{B}^{2}}{B^{4}}\right\rangle+D\left\langle\frac{1}{B^{4}}\right\rangle .
$$

Thus, an energy balance will be present if the right-hand side of Eq. (44) is zero. In principle, it should then be possible to decide whether an energy balance will occur by numerical calculation of this quantity. In practice, however, the calculation of this quantity with an acceptable accuracy would be extremely time consuming, due to the factor $1 / B^{4}$ growing fast close to the pseudocollapse, and due to the necessity of taking extremely small time steps in the integration procedure close to the singularity at $B=0$. Consequently, we have not been able to establish the energy balance numerically. However, it is interesting to note that if we make the assumption that the two quantities $1 / B^{4}$ and $\dot{B}^{2}$ are statistically independent, a sufficient condition to get balance between input and dissipation would be that

$$
\left\langle\dot{B}^{2}\right\rangle=D / \Gamma
$$

To calculate the left-hand side in (45) accurately close to pseudocollapse is also a difficult numerical task. However, if we in addition assume that the pseudocollapse process is self-averaging, we can replace the ensemble average over the pseudocollaping trajectories with a time average for one single trajectory. The following scaling behavior has then been found numerically:

$$
\lim _{t \rightarrow \infty} \frac{1}{t} \int_{t_{0}}^{t} \dot{B}^{2}\left(t^{\prime}\right) d t^{\prime} \sim \frac{D}{\Gamma}
$$

where $t_{0}$ is chosen so that $B\left(t_{0}\right)$ is small enough to be in the asymptotic regime and the proportionality constant is close to one. This relation can also be obtained using the transformation (40), from which we find

$$
\left(\frac{d B}{d t}\right)^{2}=\frac{D}{\Gamma} \frac{1}{\widetilde{D}}\left(\frac{d \widetilde{B}}{d \tilde{t}}\right)^{2}
$$

Assuming that the asymptotic behavior of the pseudocollapsing trajectories will not be affected by changing the initial condition $\widetilde{B}(0)$, the scaling relation (46) follows.

Noticing that the diverging trajectories give no contribution to the mean value (44) as $t \rightarrow \infty$, our results thus indicate that an asymptotic balance between energy input and dissipation may occur. However, we stress that we have no real justification for making the assumptions of statistical independence and self-averaging used to obtain this result.

\section{CONCLUSIONS}

We have used the method of collective coordinates to study the influence of noise and nonlinear damping on the collapse process in the two-dimensional nonlinear Schrödinger equation. This model has been shown to result under certain approximations in the description of a coupled exciton-phonon system where thermal fluctuations are taken into account. We find that the main effect of the damping term is to replace the abrupt collapse process, where the solution ceases to exist after a certain time, with a physically more reasonable exponential decrease of the width as $t \rightarrow \infty$. Concerning the influence of the noise, we have found that if the variance is large enough the wave packet may disperse instead of contracting. The critical variance necessary to cause dispersion will not be affected significantly compared to the undamped system, unless the damping is large. In the latter case, the critical variance is increased.

A comparison of the predictions obtained by the collective coordinate method to results obtained by direct numerical simulations of the partial differential equation (15) would be of interest. We note, however, that in the presence of nonlinear damping a commonly used numerical scheme such as the split-step Fourier method cannot be directly applied. It is also of interest to compare the results for the continuous equation (15) with results for the corresponding discrete equation (11). Work in this direction is in progress [12]. 


\section{ACKNOWLEDGMENTS}

We would like to thank J. Juul Rasmussen for helpful discussions. Yu.B.G. acknowledges a Guest Professorship at the Technical University of Denmark and the support of
Ukrainian Government and International Science Foundation under Grant No. 59100. M.J. acknowledges financial support from the Swedish Foundation for International Cooperation in Research and Higher Education.
[1] G. C. Huth, F. Gutmann, and G. Vitiello, Phys. Lett. A 140, 339 (1989).

[2] J. Juul Rasmussen and K. Rypdal, Phys. Scr. 33, 481 (1986).

[3] O. Bang, P. L. Christiansen, F. If, K. Ø. Rasmussen, and Yu. B. Gaididei, Phys. Rev. E 49, 4627 (1994).

[4] K. Ф. Rasmussen, Yu. B. Gaididei, O. Bang, and P. L. Christiansen, Phys. Lett. A 204, 121 (1995).

[5] G. P. Agrawal and G. Headley, Phys. Rev. A 46, 1573 (1992); Yu. S. Kivshar and S. K. Turitsyn, ibid. 47, R3502 (1993).

[6] L. Bergé, J. Juul Rasmussen, and J. Wyller, J. Phys. A (to be published).

[7] O. Bang, P. L. Christiansen, F. If, K. Ф. Rasmussen, and Yu. B. Gaididei, Appl. Anal. 57, 3 (1995).

[8] D. Anderson, M. Bonnedal, and M. Lisak, Phys. Fluids 22, 1838 (1979); M. Desaix, D. Anderson, and M. Lisak, J. Opt.
Soc. Am. 8, 2082 (1991).

[9] In fact, a Painlevé analysis of Eq. (15) shows that if the choice $f(x)=\operatorname{sech}(x)$ is made, the corresponding $\psi$ will not have the correct singularity structure to be an exact solution when $\Lambda \neq 0$. In this respect, the choice $f(x)=[\operatorname{sech}(x)]^{1 / 2}$ would be more appropriate in the presence of damping. We thank the referee for this remark.

[10] L. Lapidus and J. H. Seinfeld, Numerical Solution of Ordinary Differential Equations (Academic, New York, 1971), and references therein.

[11] T. C. Gard, Introduction to Stochastic Differential Equations (Marcel Dekker, New York, 1988), and references therein.

[12] P. L. Christiansen, Yu. B. Gaididei, M. Johansson, and K. Ø. Rasmussen (unpublished). 